\title{
Minimal resonator loss for circuit quantum electrodynamics
}

\author{
R. Barends, ${ }^{1}$ N. Vercruyssen, ${ }^{1}$ A. Endo, ${ }^{1}$ P. J. de Visser, ${ }^{1,2}$ T. Zijlstra, ${ }^{1}$ T. M. Klapwijk, ${ }^{1}$ \\ P. Diener, ${ }^{2}$ S. J. C. Yates, ${ }^{2}$ and J. J. A. Baselmans ${ }^{2}$ \\ ${ }^{1}$ Kavli Institute of NanoScience, Faculty of Applied Sciences, Delft University of Technology, Lorentzweg 1, \\ 2628 CJ Delft, The Netherlands \\ ${ }^{2}$ SRON Netherlands Institute for Space Research, Sorbonnelaan 2, 3584 CA Utrecht, The Netherlands
}

(Received 3 May 2010; accepted 9 June 2010; published online 13 July 2010)

\begin{abstract}
We report quality factors of up to $500 \times 10^{3}$ in superconducting resonators at the single photon levels needed for circuit quantum electrodynamics. This result is achieved by using NbTiN and removing the dielectric from regions with high electric fields. As demonstrated by a comparison with Ta, the crucial sources of intensity-dependent loss are dielectrics on the surface of the metal and substrate. (C) 2010 American Institute of Physics. [doi:10.1063/1.3458705]
\end{abstract}

In circuit quantum electrodynamics quantum information processing is done by coupling the qubit state to a single photon bound to a superconducting resonator. ${ }^{1}$ The lifetime of a single photon with frequency $f$ is defined by: $\tau=Q / 2 \pi f,{ }^{2}$ therefore a high resonator quality factor $(Q)$ is needed to maximize the lifetime. Presently used resonators, made from $\mathrm{Nb}$ or $\mathrm{Al}$, have quality factors on the order of $10^{4}$ to $10^{5} \cdot{ }^{2-6}$ In contrast, superconducting resonators for astronomical photon detection ${ }^{7}$ have shown quality factors in excess of a million in the many-photon regime. One would like to maintain these high values down to the single photon level. Therefore, we study the unloaded quality factor of $\mathrm{Nb}$ $\mathrm{TiN}$ and, for comparison, Ta quarterwave resonators down to the single photon level. NbTiN has a minimal dielectric layer compared to $\mathrm{Nb}, \mathrm{Al}$ and $\mathrm{Ta}^{8}{ }^{8}$ We find that in the single photon regime the quality factor of NbTiN resonators is so high that the loss is largely due to the exposed substrate. In contrast, for Ta resonators the quality factor is limited by the metal surface. We show that a further reduction in the loss in $\mathrm{NbTiN}$ resonators is achieved by removing the substrate from the regions with a high electric field density. This increases the quality factor to half a million for resonators with a central line width of $6 \mu \mathrm{m}$, three times higher than recently reported for $\mathrm{Re}^{2}$

We use NbTiN and Ta quarterwave coplanar waveguide resonators which are capacitively coupled to a feedline, ${ }^{7,8}$ see inset Fig. 1. This allows extracting the unloaded quality factor from the feedline transmission. The NbTiN films, 300 and $50 \mathrm{~nm}$ thick, are dc sputter deposited on a hydrogen passivated high resistivity $(>1 \mathrm{k} \Omega \mathrm{cm})\langle 100\rangle$-oriented $\mathrm{Si}$ wafer. The NbTi target used contains 70 at. $\% \mathrm{Nb}$ and 30 at. \% Ti. Patterning is done by reactive ion etching in an $\mathrm{SF}_{6} / \mathrm{O}_{2}$ plasma. For the $300 \mathrm{~nm}$ thick film the critical temperature is $T_{c}=14.7 \mathrm{~K}$, the low temperature resistivity is $\rho$ $=161 \mu \Omega \mathrm{cm}$ and residual resistance ratio $R R R=0.94$. For the $50 \mathrm{~nm}$ thick film: $T_{c}=13.6 \mathrm{~K}, \rho=142 \mu \Omega \mathrm{cm}$, and $R R R=0.96$. The $150 \mathrm{~nm}$ thick $\mathrm{Ta}$ film $\left(T_{c}=4.43 \mathrm{~K}, \rho\right.$ $=8.4 \mu \Omega \mathrm{cm}$, and $R R R=3.0)$ is sputtered on a similar wafer and patterned in a $\mathrm{CF}_{4} / \mathrm{O}_{2}$ plasma. The devices are cooled to $310 \mathrm{mK}$ using a He-3 sorption cooler, and down to $60 \mathrm{mK}$ using an adiabatic demagnetization refrigerator. The sample space is magnetically shielded. ${ }^{9}$ We use a vector network analyzer, locked to a frequency standard. An isolator is placed in front of the low noise amplifier.

The unloaded quality factor of NbTiN and Ta resonators is plotted versus applied photon number ${ }^{10}$ in the resonator in
Fig. 1. The resonators have resonance frequencies in the 3-6 $\mathrm{GHz}$ range, a central line width of $S=3 \mu \mathrm{m}$ and a gap width of $W=2 \mu \mathrm{m}$. Bath temperatures are 60 and $310 \mathrm{mK}$. In the many-photon regime, quality factors between $0.8 \times 10^{6}$ and $1.5 \times 10^{6}$ are observed for both materials. In addition, in this regime the 60 and $310 \mathrm{mK}$ data overlap. With decreasing applied photon number the quality factors decrease. For $\mathrm{NbTiN}$ resonators, a weak intensity dependence is observed and quality factors decrease to $\sim 250 \times 10^{3}$ at $60 \mathrm{mK}$ in the single photon regime. On the other hand, Ta quality factors degrade quickly, decreasing to $\sim 40 \times 10^{3}$. Interestingly, an inflection point is visible in the NbTiN data around $\left\langle n_{\text {photons }}\right\rangle=10^{2}-10^{3}$, whereas Ta data show a plateau at low intensities. Additionally, at low intensities a temperature and frequency dependence develops for both materials. At 310 $\mathrm{mK}$ (open symbols) the quality factors are increased, for resonators with lower frequencies the increase is larger.

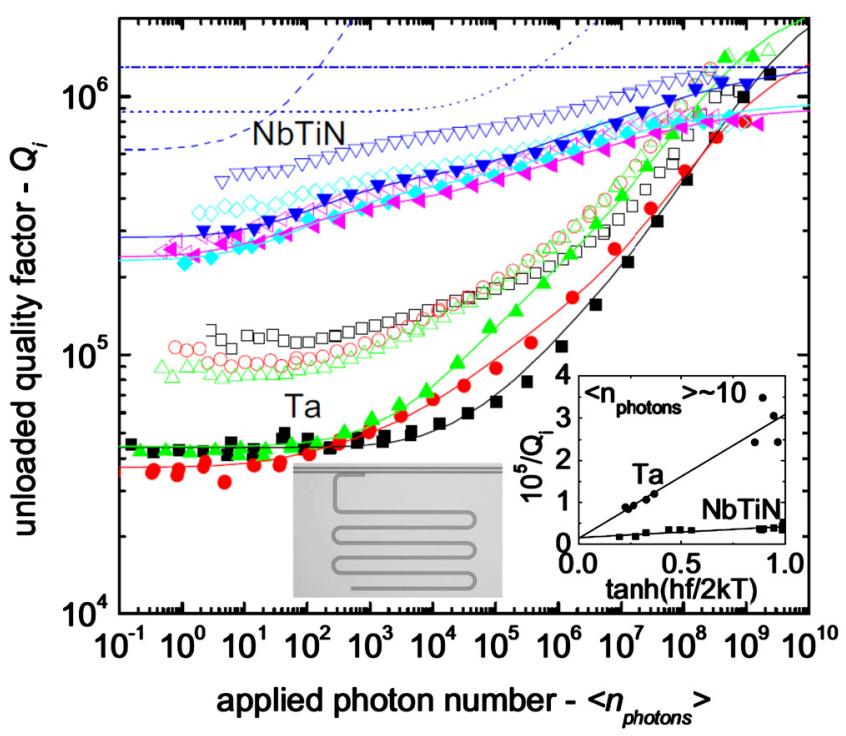

FIG. 1. (Color online) The unloaded quality factor of NbTiN and Ta quarterwave resonators vs applied microwave photon number in the resonator. Bath temperatures are $60 \mathrm{mK}$ (closed symbols) and $310 \mathrm{mK}$ (open symbols). Central line width is $S=3 \mu \mathrm{m}$ and gap width is $W=2 \mu \mathrm{m}$. Frequencies of the resonators used are $3.7(\boldsymbol{\nabla}), 4.2(\bullet)$, and $6.2(\boldsymbol{\bullet}) \mathrm{GHz}$ for the NbTiN, and $3.2(\boldsymbol{\square}), 4.5(\mathbf{O})$, and $5.0(\mathbf{\Delta}) \mathrm{GHz}$ for Ta. The solid lines are fits using Eq. (1). The quality factors of the metal surfaces (dashed), exposed substrate surface (dotted) and a fixed loss term (dashed-dotted) are shown for the 3.7 $\mathrm{GHz}$ NbTiN data. The right inset shows the microwave loss in the single photon regime vs $\tanh (h f / 2 k T)$. The left inset shows the resonator geometry. 
Previously, we have shown that NbTiN resonators contain fewer dipole two-level systems (TLS) than Ta, by measurements of the resonator frequency temperature dependence. ${ }^{8}$ Dipole TLS are configurational defects with dipole moment $p$ which reside in amorphous dielectrics, ${ }^{11}$ such as native oxides. Dielectric loss at low temperatures $(k T<h f)$ arises from resonant absorption: $1 / Q$ $\propto \tanh (h f / 2 k T) / \sqrt{1+E^{2} / E_{s}^{2}} .11,12$ The factor $\tanh (h f / 2 k T)$ reflects the thermal population difference between the lower and upper level. With increasing intensity TLS are excited, lowering the loss. The saturation field $E_{s}=\hbar / p \sqrt{T_{1} T_{2}}$ is controlled by the dipole moment and relaxation times $T_{1}$ and $T_{2}$.

The microwave loss of our resonators in the single photon regime scales with $\tanh (h f / 2 k T)$, see the inset of Fig. 1, consistent with resonant absorption from TLS. This also explains the frequency dependence which develops at $310 \mathrm{mK}$. Moreover, different resonators made from the same material follow the same trend, indicating that the loss is very comparable over the whole chip. In addition, the slope for Ta is steeper than for NbTiN, consistent with a larger TLS density for Ta, compared to NbTiN resonators.

In order to identify the location of these TLS and quantify the influence of their saturation on the quality factor, we calculate the effect of a hypothetical thin dielectric layer with thickness $t \rightarrow 0$ containing TLS. Dielectric loss in a quarterwave resonator due to dipole TLS is given $b^{2,13}$

$$
\frac{1}{Q_{\mathrm{TLS}}}=\frac{\tanh \left(\frac{h f}{2 k T}\right)}{Q_{\mathrm{TLS}, 0}} \frac{\frac{1}{2} \epsilon_{0} \epsilon_{h} \iiint_{V_{h}} \frac{|\vec{E}(\vec{r})|^{2}}{\sqrt{1+|\vec{E}(\vec{r})|^{2} / E_{s}^{2}}} d \vec{r}}{\frac{1}{4} C V_{r}^{2} l},
$$

with $V_{r}$ the standing wave voltage inside the resonator, $l$ its length, and $C$ the capacitance per unit length. The dielectric loss of the layer is $1 / Q_{\mathrm{TLS}, 0}=N \pi p^{2} / 3 \epsilon_{0} \epsilon_{h}$, with $N$ the TLS density of states and $V_{h}$ and $\epsilon_{h}$ the volume and relative permittivity of the dielectric layer hosting the TLS.

The electric fields for our resonator geometry are calculated by using the potential matrix $\mathbf{P}$ to find the charge density $q: \mathbf{V}=\mathbf{P q}{ }^{2,14}$ The substrate is included using the method of partial image charges. The potential matrix elements are given by $P_{i j}=P_{j i}=-\left(\ln \left|r_{i}-r_{j}\right|\right.$ $\left.+K \ln \left|r_{i}^{*}-r_{j}\right|\right) / 2 \pi \epsilon_{0}$ for $i \neq j$, and $P_{i i}=-\left[\ln a+K \ln \left(\left|r_{i}-r_{i}^{*}\right|\right.\right.$ $+a)] / 2 \pi \epsilon_{0}$, with $r_{i}$ the location of the $i$ th element, $r_{i}^{*}$ the location of the $i$ th element mirrored in the plane of the substrate surface, $a$ its radius, and $K=\left(1-\epsilon_{s}\right) /\left(1+\epsilon_{s}\right)$ and $\epsilon_{s}$ the relative permittivity of the substrate. The electric fields and magnetic fields are shown in Fig. 2(a).

We place this hypothetical layer on either the exposed substrate surface, top metal surface, etched metal edges, or at the substrate-metal interface. Interestingly, when the dielectric layer is placed on any of the metal surfaces, its contribution to the loss is two orders of magnitude larger than when placed on the exposed substrate [Fig. 2(c)]. This is due to the high electric fields near the metal. In addition, a dielectric placed near the metal leads to a much stronger power dependence than when located on the substrate [Fig. 2(b)]. We use this to distinguish between surfaces. Furthermore, the quality factor increases with central line width, irrespective of the location of the dielectric.

In Fig. 1, we show that the power dependence of the quality factor arises from the superposition of loss (solid

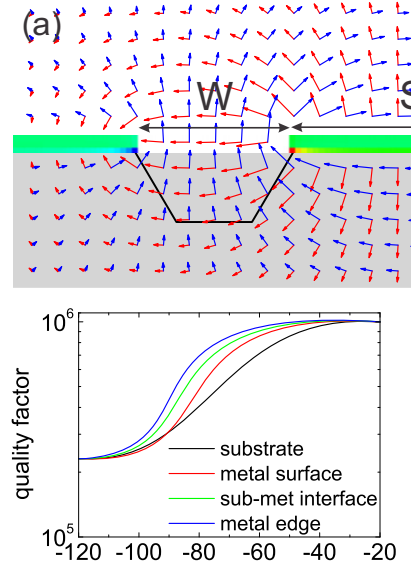

(b) internal resonator power $(\mathrm{dBm})$

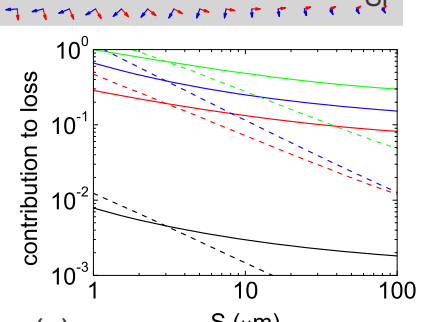

(c)
FIG. 2. (Color online) (a) The charge distribution (red denotes a positive charge, blue a negative, and green a neutral one), electric fields (red arrows), and magnetic fields (blue arrows) in the coplanar waveguide geometry. (b) The power dependence using Eq. (1) for a TLS distribution placed on the exposed substrate surface, top metal surface, substrate-metal (sub-met) interface and etched metal edges. $Q_{0}=10^{6}$, and at low intensity each surface is assumed to limit the $Q$ to $300 \times 10^{3}$. (c) The normalized contribution to loss of the dielectric layers vs central line width $S$, for $W=2 \mu \mathrm{m}$ (solid) and $W=(2 / 3) S$ (dashed)

lines) from TLS [Eq. (1)] located at the metal surfaces (dashed line) as well as at the exposed substrate surface (dotted line). ${ }^{15}$ Interestingly, for NbTiN resonators the exposed substrate, together with the metal surfaces, is a significant contributor to the microwave loss. This superposition of loss closely describes the observed point of inflection at $\left\langle n_{\text {photons }}\right\rangle=10^{2}-10^{3}$ as well.

The saturation fields of NbTiN are on the order of 50 $\mathrm{V} / \mathrm{m}$, see Table I, similar to values for Re and $\mathrm{Al}^{2}{ }^{2}$ For Ta we find a large spread in the saturation fields. The dielectric loss of NbTiN is clearly smaller than that of Ta. The substrate surface values are consistent with $\mathrm{SiO}_{x}$. The saturation field is $E_{s} \sim 2-5 \mathrm{kV} / \mathrm{m}$; comparable to measurements on vitreous silica. ${ }^{16}$ Moreover, a value of $E_{s} \sim 2-3 \mathrm{kV} / \mathrm{m}$ has been reported for $\mathrm{SiO}_{2}$ also by Martinis et al. ${ }^{12}$ The quality factor of the Si surface layer, assuming $t=3 \mathrm{~nm}$ and $\epsilon_{h}=4$, lies around 15-200, which is on the order of the value of $\sim 200$ reported for $\mathrm{SiO}_{2} .{ }^{12}$ At high intensity the quality factors are temperature independent, suggesting loss other than due to TLS. We include an intensity-independent fitting term $1 / Q_{0}$ to account for this loss. We suspect that we reach the level of the intrinsic loss of the superconductor. For Ta, relaxation times saturate for $T / T_{c}<0.2,{ }^{17}$ suggesting that the quasiparticle density becomes temperature independent. At $T / T_{c}=0.2$ we estimate

TABLE I. The quality factor of the dielectric layer containing TLS, its saturation field, and the additional loss factor for the superconducting metals and for their Si substrates, used for fitting the data in Fig. 1, using Eq. (1) and $1 / Q=1 / Q_{0}+1 / Q_{\mathrm{TLS}, \text { met }}\left(E_{s, \mathrm{met}}\right)+1 / Q_{\mathrm{TLS}, \mathrm{sub}}\left(E_{s, \text { sub }}\right)$. Calculations have been done for $\epsilon_{h}=1$ and $t \rightarrow 0$.

\begin{tabular}{lccc}
\hline \hline \multicolumn{1}{c}{ Material } & $Q_{0}\left(10^{6}\right)$ & $\begin{array}{c}Q_{\mathrm{TLS}, 0} / \epsilon_{h} t \\
(1 / \mathrm{nm})\end{array}$ & $\begin{array}{c}E_{s} \\
(\mathrm{kV} / \mathrm{m})\end{array}$ \\
\hline $\mathrm{NbTiN}$ & $0.9-1.3$ & $330-450$ & 0.05 \\
$\mathrm{Ta}$ & $1.7-3$ & $70-90$ & $0.1-2$ \\
$\mathrm{Si}(\mathrm{NbTiN})$ & & $13-16$ & 5 \\
$\mathrm{Si} \mathrm{(Ta)}$ & & $1.1-1.9$ & 2 \\
\hline \hline
\end{tabular}




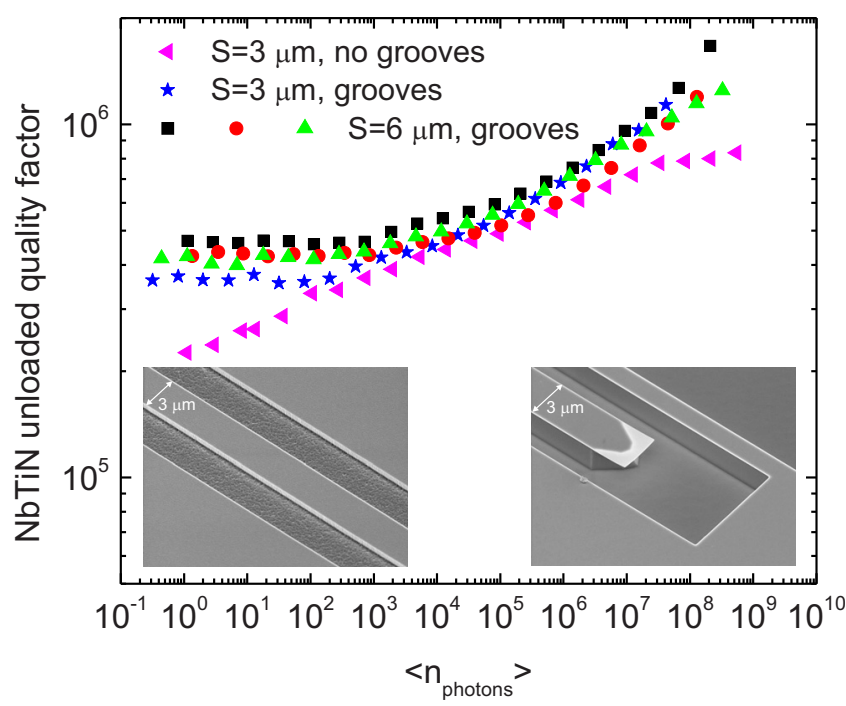

FIG. 3. (Color online) The unloaded quality factor vs applied photon number of NbTiN quarterwave resonators with the standard geometry and $S$ $=3 \mu \mathrm{m}$ and $W=2 \mu \mathrm{m}(4)(6.2 \mathrm{GHz})$, and with grooves etched in the exposed Si substrate with $S=3 \mu \mathrm{m}$ and $W=2 \mu \mathrm{m}(\star)(4.7 \mathrm{GHz})$, and $S$ $=6 \mu \mathrm{m}$ and $W=2 \mu \mathrm{m}$ at frequencies of $4.2(\boldsymbol{\square}), 4.4(\bullet)$, and $5.2(\boldsymbol{\Delta}) \mathrm{GHz}$. Bath temperature is $60 \mathrm{mK}$. The left inset is a scanning electron microscope image from the standard coplanar waveguide design, the right inset shows the etched grooves near the open end of the resonator $(S=3 \mu \mathrm{m}$ in both images). The cross section of the etched resonators is outlined in Fig. 2(a).

$Q \sim 10^{6}$ based on Mattis-Bardeen, on the order of values found for $Q_{0}$.

The data in Fig. 1 and the analysis provide a clear guide toward improving the quality factor. Ta suffers from significant microwave loss due to dipole TLS in its metal surface. We believe that the presence of a native oxide is the reason why resonators made of $\mathrm{Nb}, \mathrm{Ta}, \mathrm{Al}$, or deposited on top of $\mathrm{SiO}_{2}$, consistently show low quality factors in the single photon regime. ${ }^{2-6}$ In this respect NbTiN is different because the metal atoms are bound to nitrogen. Moreover, resonators with $S=3 \mu \mathrm{m}$ and $W=2 \mu \mathrm{m}$ have quality factors around $250 \times 10^{3}$, nearly a doubling compared to Re on Si resonators which have quality factors around $150 \times 10^{3}$ (loaded, with $\left.Q_{c}>Q_{i}\right)$ and are wider $(S=5 \mu \mathrm{m}$ and $W=2 \mu \mathrm{m}){ }^{2}$ Nevertheless, the NbTiN resonator quality factor is significantly limited by the exposed $\mathrm{Si}$ surface. Therefore NbTiN has a clean surface compared to $\mathrm{Si}$, as the metal influences the loss much stronger [Fig. 2(c)].

With NbTiN shown to be a superior superconductor, we have redesigned our resonators to have fewer dielectrics. We have made $50 \mathrm{~nm}$ thick NbTiN resonators, fully straight, which are aligned along the $\langle 110\rangle$ axis of the $\langle 100\rangle$-oriented HF-cleaned Si wafer. Using $\mathrm{KOH}$ wet etching, grooves of $0.9 \mu \mathrm{m}$ deep are etched in the gaps along the full length of the resonators, see the inset of Fig. 3; this removes the substrate surface from the region with the highest electric field density [black lines in Fig. 2(a)].

The NbTiN resonators with grooves etched in the gaps have significantly higher quality factors, see Fig. 3. In the single photon regime, the quality factor has improved from a value of $250 \times 10^{3}$ for the standard design to an intensityindependent plateau value of $350 \times 10^{3}$ for the etched resonators, for $S=3 \mu \mathrm{m}$ and $W=2 \mu \mathrm{m}$. Moreover, this increase is a clear indication that the Si surface was the limiting factor also for another reason: the decrease in dielectric has lead to a decrease in the capacitance $C$ in Eq. (1). Therefore, if the metal surfaces would dominate the losses, the quality factors would decrease. The intensity-independent plateau points towards a single surface dominated loss. With the Si removed, the loss at the single photon level is dominated by the metal surfaces. Determining which surface is complicated by the similarity in dependence on intensity and width (Fig. 2). In the many-photon regime the loss is more due to the exposed substrate surface, indicated by the higher quality factors for etched resonators and the high saturation field values. When increasing the width to $S=6 \mu \mathrm{m}$ and $W=2 \mu \mathrm{m}$, the quality factor improves to around $450 \times 10^{3}$. This increase is consistent with Fig. 2(b) and shows that further increases can be obtained by widening.

With quality factors as high as $470 \times 10^{3}$, we estimate single photon lifetimes of $18 \mu \mathrm{s}$ at $4.2 \mathrm{GHz}$, one order of magnitude longer than decoherence times measured for superconducting qubits. ${ }^{18,19}$ These long lifetimes make superconducting resonators, as shown in Fig. 3, appealing building blocks for a quantum processor, as they can be used as quantum memory elements ${ }^{20}$ and for a quantum bus for longrange qubit-qubit coupling. ${ }^{21,22}$ Interestingly, the resonators with grooves also have less frequency noise. ${ }^{23}$

To conclude, we have found NbTiN resonators to have a higher quality factor in the single photon regime than any of the previously studied superconductors, indicating it has a minimal lossy dielectric layer. The losses arise largely due to a surface distribution of TLS on the exposed Si substrate. By removing the substrate from the region with highest electric fields the quality factor is increased further, showing that using NbTiN resonators and removing dielectrics is a straightforward route to high quality factors in the single photon regime.

The authors thank J. M. Martinis and P. Forn-Díaz for stimulating discussions. The work was supported by the Pieter Langerhuizen Lambertuszoon funds of the Royal Holland Society of Sciences and Humanities and by the EU NanoSciERA project "Nanofridge."

\footnotetext{
${ }^{1}$ A. Wallraff et al., Nature (London) 431, 162 (2004).

${ }^{2}$ H. Wang et al., Appl. Phys. Lett. 95, 233508 (2009).

${ }^{3}$ A. D. O'Connell et al., Appl. Phys. Lett. 92, 112903 (2008).

${ }^{4}$ P. Macha et al., Appl. Phys. Lett. 96, 062503 (2010).

${ }^{5}$ T. Lindström et al., Phys. Rev. B 80, 132501 (2009).

${ }^{6}$ A. Palacios-Laloy et al., J. Low Temp. Phys. 151, 1034 (2008)

${ }^{7}$ P. K. Day et al., Nature (London) 425, 817 (2003).

${ }^{8}$ R. Barends et al., Appl. Phys. Lett. 92, 223502 (2008).

${ }^{9}$ R. Barends, Ph.D. thesis, Delft University of Technology, 2009.

${ }^{10}$ Photon number: $\left\langle n_{\text {photons }}\right\rangle=C V_{r}^{2} l / 2 h f$, with $V_{r}=2 \sqrt{P_{\text {int }} Z}$ the standing wave voltage, $P_{\text {int }}$ the internal power, ${ }^{9}$ and $Z$ the impedance.

${ }^{11}$ W. A. Phillips, Rep. Prog. Phys. 50, 1657 (1987).

${ }^{12}$ J. M. Martinis et al., Phys. Rev. Lett. 95, 210503 (2005).

${ }^{13}$ J. Gao et al., Appl. Phys. Lett. 92, 152505 (2008).

${ }^{14}$ P. P. Silvester and R. L. Ferrari, Finite Elements for Electrical Engineers, 2nd ed. (Cambridge University Press, Cambridge, U.K., 1989).

${ }^{15}$ At $T>h f / k$ absorption due to relaxational interaction can become relevant as well. ${ }^{11}$ We therefore limit the use of Eq. (1) to the $60 \mathrm{mK}$ data.

${ }^{16}$ M. von Schickfus and S. Hunklinger, Phys. Lett. 64A, 144 (1977).

${ }^{17}$ R. Barends et al., Phys. Rev. Lett. 100, 257002 (2008).

${ }^{18}$ P. Bertet et al., Phys. Rev. Lett. 95, 257002 (2005).

${ }^{19}$ A. A. Houck et al., Phys. Rev. Lett. 101, 080502 (2008).

${ }^{20}$ P. J. Leek et al., Phys. Rev. Lett. 104, 100504 (2010).

${ }^{21}$ M. A. Sillanpää et al., Nature (London) 449, 438 (2007).

${ }^{22}$ J. Majer et al., Nature (London) 449, 443 (2007).

${ }^{23} \mathrm{R}$. Barends et al., arXiv:1005.5394.
} 